\title{
Ultrafast dynamics of photoprocesses induced by femtosecond IR laser radiation in iron pentacarbonyl molecules and clusters
}

\author{
D.G. Poydashev*, V.O. Kompanets, V.N. Lokhman, S.V. Chekalin, and E.A. Ryabov \\ Institute for Spectroscopy RAS, 108840 Troitsk, Moscow, Russia
}

\begin{abstract}
Ultrafast dynamics of photoinduced processes in $\left[\mathrm{Fe}(\mathrm{CO})_{5}\right]_{n}$ clusters and free $\mathrm{Fe}(\mathrm{CO})_{5}$ molecules excited by resonant femtosecond IR radiation $(\lambda \sim 5 \mu \mathrm{m})$ were studied in a molecular beam. Using a pump-probe teqnique characteristic rates of these processes were obtained and theoretically analyzed.
\end{abstract}

The interaction of resonant femtosecond IR radiation with $\mathrm{CO}$ vibrational modes of both free $\mathrm{Fe}(\mathrm{CO})_{5}$ molecules and $\left[\mathrm{Fe}(\mathrm{CO})_{5}\right]_{n}$ molecular clusters followed by subsequent photoinduced processes are the main subject of the studies presented in this report. The experiments were carried out using a combination of femtosecond laser spectroscopy and photoionization timeof-flight mass spectrometry (TOF MS) techniques. The clusters and cold free molecules were produced by supersonic expansion of $\mathrm{Fe}(\mathrm{CO})_{5}+$ Ar mixture from a pulsed nozzle.

The technique of the multiphoton ionization of molecules by femtosecond ultraviolet or visible radiation in combination with time-of-flight mass spectrometry has been used for the first time to probe the dynamics of processes in vibrationally excited molecules in a molecular beam. It has been found that the resonant excitation of vibrations of $\mathrm{CO}$ bonds in free $\mathrm{Fe}(\mathrm{CO})_{5}$ molecules below their dissociation threshold results in an "instantaneous" (on a time scale of $\approx 800 \mathrm{fs}$ ) significant decrease in the signal from the $\mathrm{Fe}(\mathrm{CO})_{5}{ }^{+}$molecular ion (see Fig. 1). The subsequent time relaxation of this signal, as well as the dependence of an induced change in this signal on the energy fluence $\Phi_{\mathrm{IR}}$ of the infrared pump pulse, has been studied.

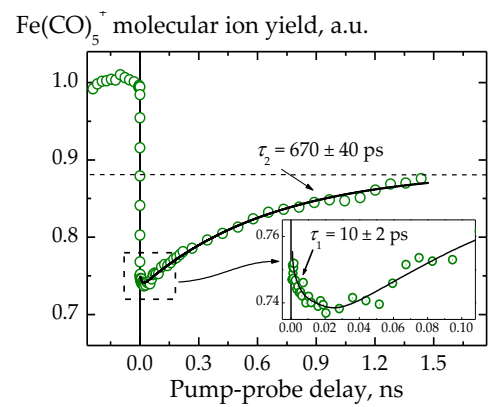

Fig. 1. Dynamics of $\mathrm{Fe}(\mathrm{CO}) 5^{+}$molecular ion yield in the case of IR excitation of free molecules.

\footnotetext{
*Corresponding author: poydashev@isan.troitsk.ru
} 
The analysis of the results indicates that the infrared pulse selectively excites vibrational modes of the $\mathrm{CO}$ bonds. A decrease in the multiphoton ionization cross section for the probe pulse as a result of this excitation leads to an initial decrease in the yield of $\mathrm{Fe}(\mathrm{CO})_{5}^{+}$ molecular ion. It has been found that this signal further relaxes with two characteristic times - short, $\tau_{1} \approx 10 \mathrm{ps}$, and long, $\tau_{2} \approx 670 \mathrm{ps}$ (see Fig. 1). Slow relaxation is due to the intramolecular relaxation of the energy localized in the vibrations of $\mathrm{CO}$ bonds to other vibrations of the molecule, and $\tau_{2}$ is the characteristic time of this process. The time $\tau_{1}$ is possibly associated with Berry pseudorotation (for details, see ref.1).

Using the femtosecond time-resolved infrared pump - visible probe technique, we have measured for the first time the ultrafast dissociation dynamics of $\left[\mathrm{Fe}(\mathrm{CO})_{5}\right]_{n}$ clusters induced by IR resonant excitation of $\mathrm{CO}$ vibrations in the $5 \mu \mathrm{m}$ region. Relaxation of vibrational energy stored by clusterized molecules leads to an increase of the temperature of the cluster and subsequent evaporation of free $\mathrm{Fe}(\mathrm{CO})_{5}$ molecules formed as a result of the cluster dissociation. These molecules have been ionized by the femtosecond laser radiation at $\lambda=$ $400 \mathrm{~nm}$ and have been detected by TOF MS device (left side of Fig. 2).

The temporal dependence of the yield of free molecules has been measured under different conditions of the IR laser excitation. Experiments with femtosecond IR radiation with different widths of the spectrum $\left(100 \mathrm{~cm}^{-1}\right.$ and $\left.185 \mathrm{~cm}^{-1}\right)$ have allowed us to conclude that the dynamics of the yield of free molecules from clusters is determined by the magnitude of the energy absorbed by the cluster, with the molecules in the cluster utilizing only the «resonant» part of the radiation spectrum.
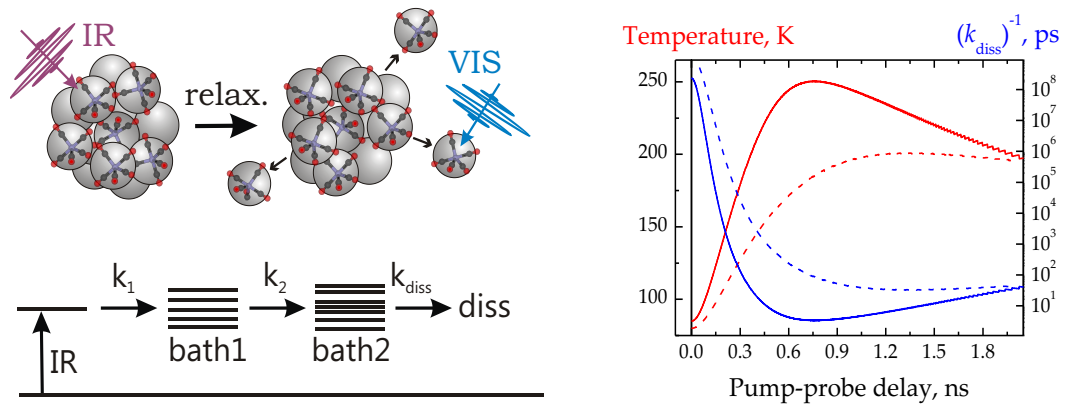

Fig. 2. Left: schematic diagram of the processes involved in the dissociation of clusters. Right: dynamic dependencies for cluster temperature $T$ and dissociation rate $k_{\text {diss }}$ in $\left[\mathrm{Fe}(\mathrm{CO})_{5}\right]_{n}$ clusters. Solid and dashed lines are calculations for $100 \mathrm{~cm}^{-1}$ and $185 \mathrm{~cm}^{-1}$ IR bandwidth respectively.

We have proposed a model that describes well experimental results and makes it possible to calculate the temporal profile of the cluster temperature as well as cluster dissociation rate $k_{\text {diss }}$ in terms of the concept of the evaporative ensemble (see right side of Fig. 2). The rates of the intramolecular and intracluster vibrational relaxation in $\left[\mathrm{Fe}(\mathrm{CO})_{5}\right]_{n}$ clusters have been estimated (for the details of the modelling see ref. 2). For instance, the model shows that the characteristic time of the initial energy relaxation from excited $\mathrm{CO}$ vibrations to lowfrequency modes of the molecule in cluster is close to the characteristic time of the subsequent vibrational energy redistribution from low-frequency modes of the molecule into the intermolecular vibrations of the cluster and equals to approx. $280 \mathrm{ps}$.

\section{References}

1. V.O. Kompanets, V.N. Lokhman, D.G. Poydashev, S.V. Chekalin, E.A. Ryabov, JETP 122, 621 (2016)

2. D.G. Poydashev, V.N. Lokhman, V.O. Kompanets, S.V. Chekalin, E.A. Ryabov, J. Phys. Chem. A 118, 11177 (2014) 